\title{
COMPARAÇÃO ENTRE RESULTADOS COMPUTACIONAIS E EXPERIMENTAIS DO COMPORTAMENTO TÉRMICO DE UM AMBIENTE
}

\author{
Edi Terezinha de Oliveira Grings ${ }^{1}$, Paulo Otto Beyer ${ }^{2}$
}

\section{RESUMO}

O presente trabalho apresenta uma comparação entre resultados computacionais, obtidos através do "software" EnergyPlus, e experimentais do comportamento térmico de um ambiente condicionado e não condicionado. Para tanto, monitoraram-se os dados climáticos de radiação, velocidade do vento e temperatura, no período de 11 a 20 de janeiro de 2002, e produziu-se um arquivo climático. Simultaneamente foi feita a aquisição das temperaturas de uma salateste, localizada no terceiro pavimento de um prédio na cidade de Porto Alegre. As temperaturas do ar de insuflamento e de retorno dos condicionadores de ar, localizados na sala, foram medidas durante o dia, em seis dias do período de monitoramento. A velocidade do ar de retorno foi coletada e calculou-se a potência sensível de refrigeração. Os resultados da carga térmica e temperatura ambiente da sala-teste obtidos nas simulações foram comparados com os dados experimentais. Houve uma boa concordância entre os resultados computacionais e experimentais.

Palavras-chave: Comportamento térmico de edificações, Simulação térmica de ambientes, EnergyPlus

\section{ABSTRACT}

This work presents a comparison between computational results, obtained through the use of the software EnergyPlus, and experimental results of the thermal behavior of an air-conditioned and non-air-conditioned environment. In order to do that, climatic data on radiation, wind speed and temperature were monitored in the period between the 11 and 20 of January 2002 and a climatic. Simultaneously, the temperatures of a test-room, situated on the 3rd floor of a building in the town of Porto Alegre, were collected. The suply and return temperatures of the air conditioners, situated in the testroom, were measured during the day, in 6 days of the monitoring period. The return air speed was measured and refrigeration sensitive capacity calculated. The results of the thermal load and the environmental temperature of the testroom obtained in the simulations were compared with the experimental data. There was a good agreement between the computational and experimental results.

Key words: Thermal behaviour of a construction, Simulation thermal of and environment, EnergyPlus.

\section{INTRODUÇÃO}

Muitos trabalhos como o de Hernandez et al., 2001, que compara resultados simulados por vários "softwares" com dados medidos e pontua que se poderia chegar a diferentes conclusões a respeito de conforto térmico, em função dos diferentes resultados dos "sofwares" utilizados, mostra a discrepância entre resultados medidos e simulados.

Diante das diferenças encontradas entre resultados computacionais e experimentais, este trabalho se propõe a avaliar a carga térmica em ambiente climatizado e a temperatura em evolução livre, encontradas através de simulação realizada com o Programa EnergyPlus e comparar com resultados medidos.

\section{MATERIAI E MÉTODOS}

A sala escolhida para teste está localizada no prédio Instituto Parobé no centro de Porto Alegre. A fachada principal do prédio forma $29^{\circ} \mathrm{com}$ o norte verdadeiro. Ela fica no terceiro pavimento de um prédio, que possui quatro andares. A figura 1 é um croqui da sala. As dimensões são dadas em metro.

As paredes da frente e dos fundos estão expostas ao ambiente externo e têm espessura de $50 \mathrm{~cm}$. A divisórias laterais medem $18 \mathrm{~cm}$.

O período definido para a realização dos ensaios foi de 11 a 20 de janeiro de 2002. Foram realizadas as medidas das temperaturas de bulbo seco e úmido da sala-teste, da entrada e da saída do evaporador

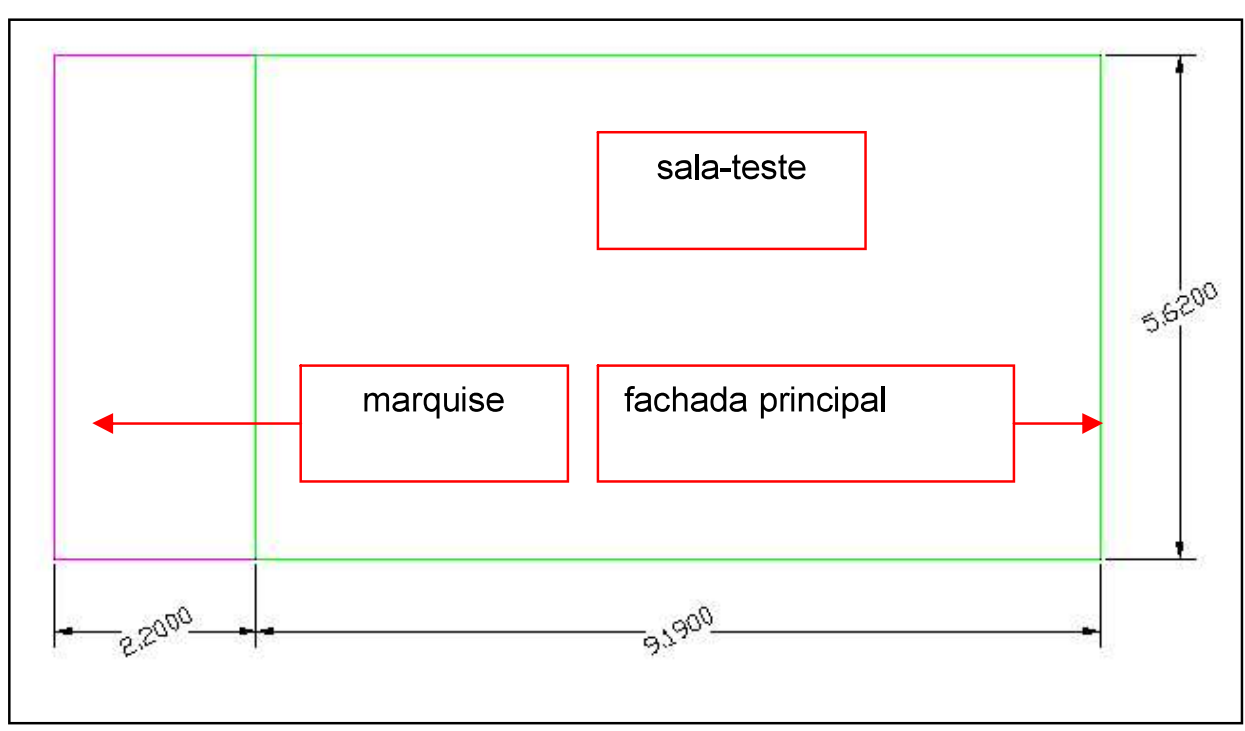

Figura 1 - Croqui da sala-teste. 
dos condicionadores de ar; temperaturas de bulbo seco e úmido externa; das temperaturas de bulbo seco das salas adjacentes; da radiação solar global e difusa; da velocidade e direção do vento; e da velocidade do ar na entrada do evaporador. A aquisição das temperaturas, radiação e velocidade do vento foram feitas simultaneamente em intervalos de $5 \mathrm{~min}$.

Para a determinação da potência sensível de refrigeração foram utilizados dois condicionadores de ar instalados na sala. $\mathrm{Na}$ entrada e na saída do evaporador dos condicionadores de ar foram conectados dutos, cujas áreas foram divididas em seções. No centro de cada seção foram medidas as velocidades do ar. Para o cálculo da potência sensível de refrigeração foram utilizadas as vazões e temperaturas medidas.

Foi organizado um arquivo climático específico para o período em que foi feita a análise do desempenho térmico da sala-teste, com o objetivo de informar ao EnergyPlus as condições climáticas reais dos dias em que as potências de refrigeração foram medidas. Os dados coletados foram organizados num arquivo com dados horários e são mostrados na figura 2 .

\section{Variáveis Climáticas Medidas}

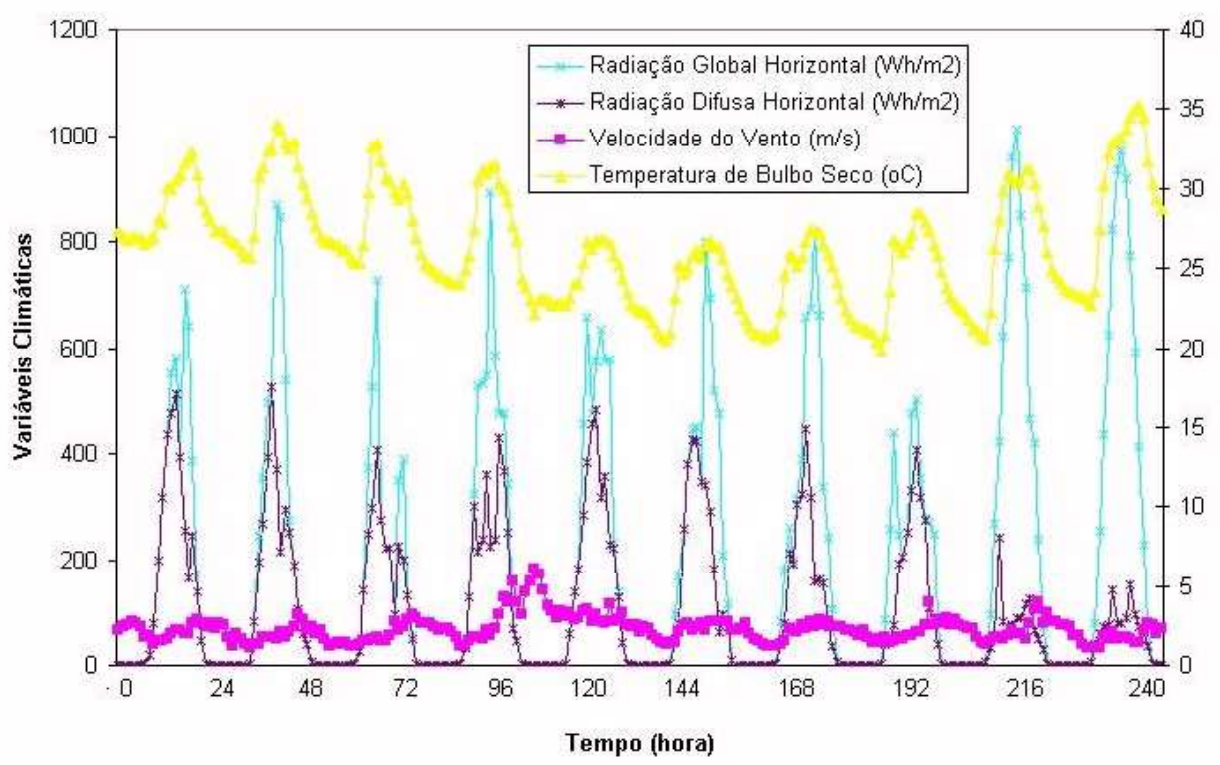

Figura 2 - Variáveis climáticas medidas.

A simulação foi implementada através da declaração de dados de entrada. As características termofísicas dos materiais foram definidas conforme mostra os dados registrados na tabela 1.

Tabela 2 - Carga térmica gerada na sala.
A carga térmica interna era gerada por lâmpadas e equipamentos cujas correntes e tensões são mostradas na tabela 2

Para estimar-se a vazão de ventilação obtida por infiltração, utilizou-se o método da área de frestas.

empo (hora)

\section{RESULTADOS}

A figura 3 mostra o gráfico das temperaturas internas da sala-teste medidas e simuladas nos dias 19 e 20 de janeiro de 2002. A sala-teste não estava condicionada. A diferença

\begin{tabular}{llllll}
\hline Nome & Tijolo & Argamassa & Lajota & Concreto & Madeira \\
\hline Tipo & Rugoso & Médio & Médio & Médio & Médio \\
Espessura $[\mathrm{m}]$ & $0,16-0,48$ & 0,01 & 0,3 & $0,035-0,010$ & 0,035 \\
Condut. $[\mathrm{W} / \mathrm{mK}]$ & 1,05 & 1,15 & 1,05 & 1,75 & 0,23 \\
Densidade $\left[\mathrm{kg} / \mathrm{m}^{3}\right]$ & 2000 & 2100 & 2000 & 2400 & 750 \\
Calor espec. $[\mathrm{J} / \mathrm{kgK}]$ & 920 & 1000 & 920 & 1000 & 1340 \\
Absortância térmica & 0,9 & 0,9 & 0,9 & 0,9 & 0,9 \\
Absot. Solar & & $0,2-0,4$ & 0,74 & & 0,3 \\
\hline
\end{tabular}

Tabela 1 - Características termofísicas dos materiais de construção

\begin{tabular}{lccc}
\hline Fonte & Tensão $(\mathrm{V})$ & Corrente $(\mathrm{A})$ & Calor gerado \\
\hline Pessoas & - & - & $99 \mathrm{~W} /$ pessoa \\
Lâmpadas & 116 & 1,15 & $798 \mathrm{~W}$ \\
Computadores e monitores & 116 & 1,22 & $425 \mathrm{~W}$ \\
Motor-ventiladores & 220 & $0,49-0,58$ & $78 \mathrm{~W}$ \\
HP-Data Acquisition & 116 & 0,08 & $9 \mathrm{~W}$ \\
Refrigerador experimental & 116 & 2,00 & $12 \mathrm{~W}$ \\
\hline
\end{tabular}

máxima entre as temperaturas medidas e simuladas, nestes dois dias, foi de 1,0 oC e a diferença média foi de 0,5 oC. Este trabalho apresenta resultados semelhantes aos encontrados pelo trabalho de Hernandez et al, 2001, que, em um dia, encontraram diferença máxima na temperatura de 1,3 oC e diferença média de 0,6 oC com o "software" NBSLD modificado para reproduzir melhor as condições climáticas brasileiras.

O gráfico, da figura 4 , mostra a carga térmica medida com a margem de incerteza prevista e a carga simulada nos dias 19 e 20 de janeiro de 2002. A sala-teste estava condicionada.

Observa-se, através do gráfico, que a diferença entre a carga térmica medida e simulada apresenta-se dentro do intervalo da incerteza prevista. A diferença máxima entre os resultados experimentais e simulados é de $288 \mathrm{~W}$ e ocorre às $10 \mathrm{~h}$ do dia 18/01. A diferença média é $74 \mathrm{~W}$.

\section{DISCUSSÃO}

No estudo comparativo realizado considerou-se que houve uma boa concordância entre as temperaturas e as cargas térmica medidas e simuladas no período.

É necessário ressaltar que, dependendo da opção do usuário do programa computacional, as diferenças 


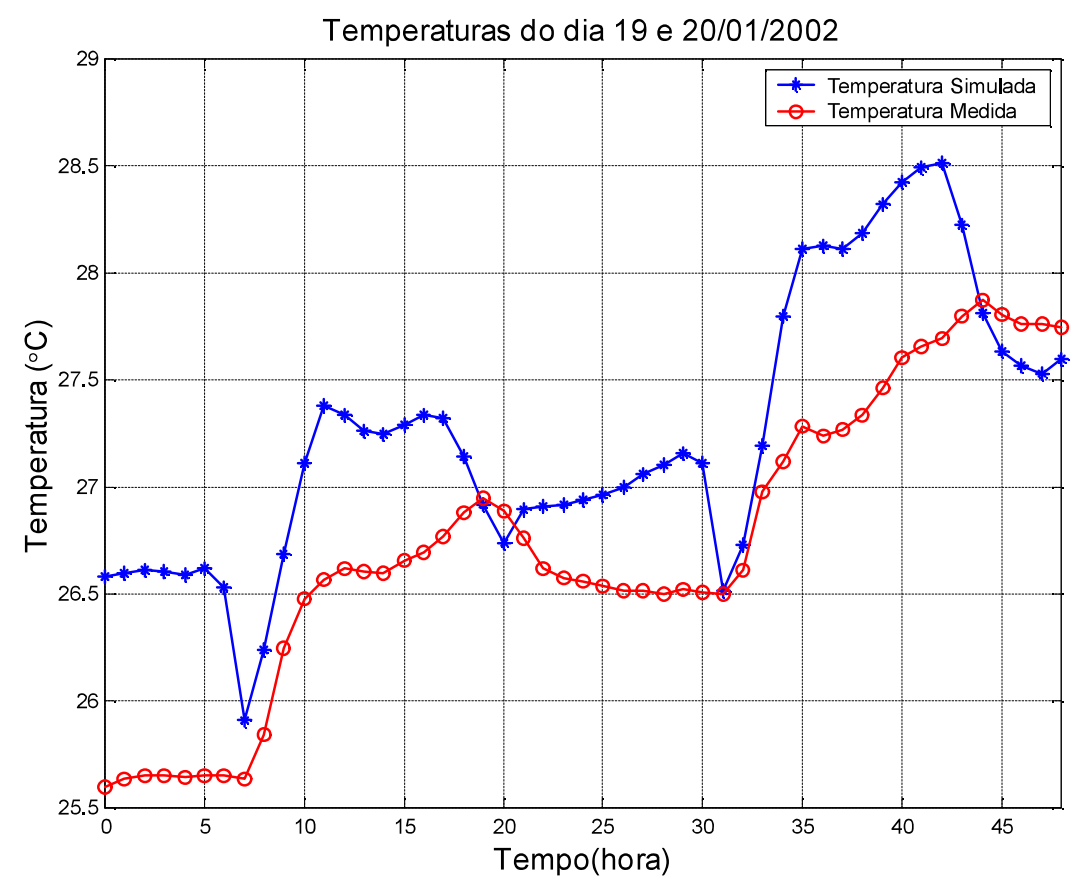

medida; a impossibilidade da entrada no programa de aberturas com forma geométrica circular; a hipótese que desconsidera 0 gradiente na temperatura do ar da zona e a utilização de equipamentos de medição simples, que limitam a precisão dos resultados.

Finalmente, merece que se diga que - EnergyPlus é uma ferramenta poderosa no campo das simulações térmicas, apresentando resultados com boa concordância, quando comparados a resultados experimentais, em simulações realizadas em edificações de elevada inércia térmica.

Figura 3 - Gráfico da temperatura da sala-teste.

entre os resultados medidos e simulados poderão ser diferentes. É importante, portanto, que se conheçam as vantagens e limitações da ferramenta computacional disponível para simulações termoenergéticas.

A infiltração é uma grandeza difícil de ser determinada ou estimada, merecendo muitas horas de simulações e ajustes até se obter um valor adequado e coerente.

Muitos fatores podem ter contribuído para as diferenças apresentadas entre os resultados medidos e simulados, tais como: as características termofísicas dos materiais, que podem não representar exatamente as que foram utilizados na construção; os dados de entrada obtidos a partir de medições que levam consigo uma incerteza da

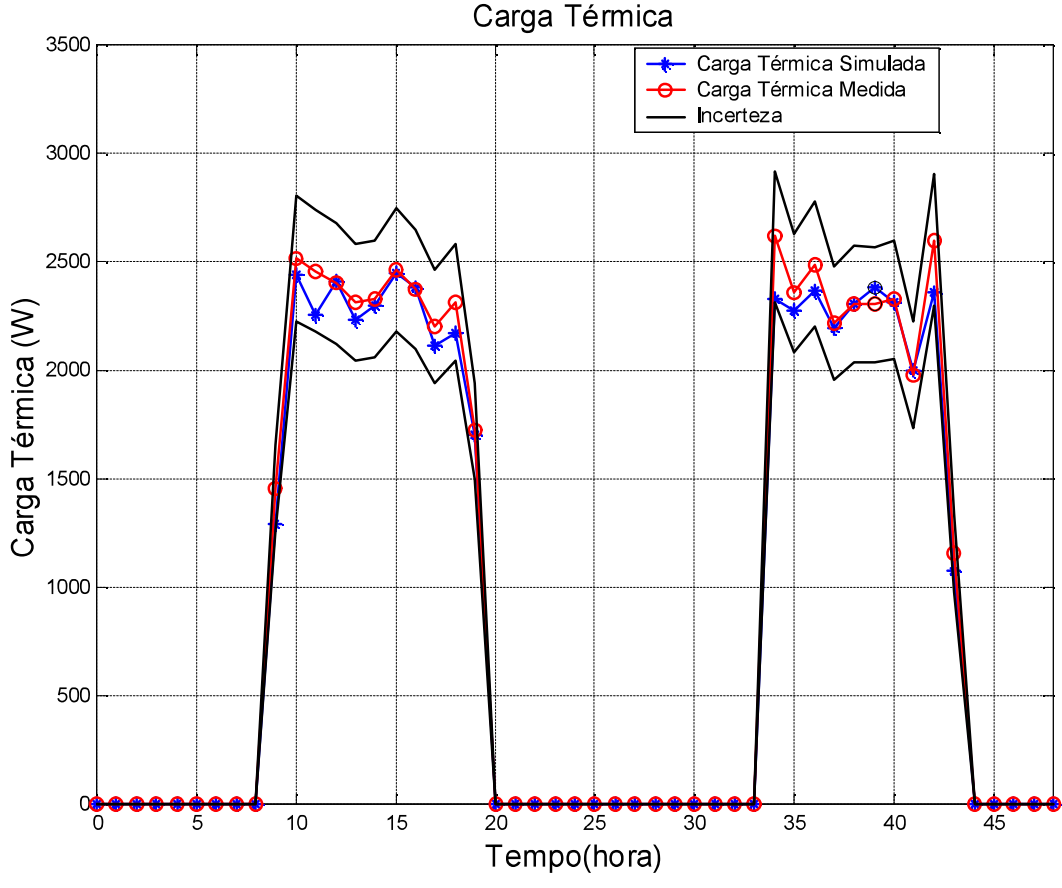

Figura 4 - Gráfico da carga térmica e incerteza.

\section{REFERÊNCIAS BIBLIOGRÁFICAS}

1. EnergyPlus Manual. COPYRIGHT 1998-2002, GARD. Version 1.0.2; July 2002.

2. Duffie JA, Beckman WA. Solar Engineering of Thermal Processes.2a ed. New York: John Wiley and Sons; 1991.

3. Hernandez AN, Tribess A, Vittorino F. Evaluation of simulation software for non-conditioned houses. XVI Congresso Brasileiro de Engenharia Mecânica; Nov 26-30; Uberlândia, Brasil; 2001. 5 vol. pp. 70-75.

4. Holman JP. Experimental Methods for Engineers. 2a ed. McGraw-Hill; 1971. 